\title{
A randomised controlled trial to compare methods of milk expression after preterm delivery
}

\author{
E Jones, P W Dimmock, S A Spencer
}

\begin{abstract}
Objectives-Primary: to compare sequential and simultaneous breast pumping on volume of milk expressed and its fat content. Secondary: to measure the effect of breast massage on milk volume and fat content.

Design-Sequential randomised controlled trial.

Setting-Neonatal intensive care unit, North Staffordshire Hospital NHS Trust. Subjects-Data on 36 women were analysed; 19 women used simultaneous pumping and 17 used sequential pumping. Interventions-Women were randomly allocated to use either simultaneous (both breasts simultaneously) or sequential (one breast then the other) milk expression. Stratification was used to ensure that the groups were balanced for parity and gestation. A crossover design was used for massage, with patients acting as their own controls. Women were randomly allocated to receive either massage or non-massage first.
\end{abstract}

Main outcome measures-Volume of milk expressed per expression and its fat content (estimated by the creamatocrit method).

Results-Milk yield per expression was: sequential pumping with no massage, $51.32 \mathrm{~g}$ (95\% confidence interval (CI) 56.57 to 46.07); sequential pumping with massage, $78.71 \mathrm{~g}$ (95\% CI 85.19 to 72.24$)$; simultaneous pumping with no massage, 87.69 g (95\% CI 96.80 to 78.57$)$; simultaneous pumping with massage, $125.08 \mathrm{~g}(95 \%$ CI 140.43 to 109.74). The fat concentration in the milk was not affected by the increase in volume achieved by the interventions.

Conclusions-The results are unequivocal and show that simultaneous pumping is more effective at producing milk than sequential pumping and that breast massage has an additive effect, improving milk production in both groups. As frequent and efficient milk removal is essential for continued production of milk, mothers of preterm infants wishing to express milk for their sick babies should be taught these techniques.

(Arch Dis Child Fetal Neonatal Ed 2001;85:F91-F95)

Keywords: lactation; milk production; preterm infants; premature

The immediate period after preterm delivery is a time of extreme anxiety for parents. The initiation of a milk expression schedule can be difficult to achieve because it may necessitate a strong commitment to the future that is impossible to make. There is an inverse relation between gestational age and breast feeding outcome. ${ }^{1-3}$ The delay in initiation, the immaturity of the mammary gland, and the inhibition of milk ejection caused by stress may result in poor milk yield and declining milk production. ${ }^{4}$ Mothers often report that the provision of their milk is the only positive contribution that they can make when health care professionals $^{56}$ undertake the care of their infant. Failure to breast feed may contribute to maternal feelings of helplessness and guilt. ${ }^{78}$

Human milk is better absorbed than formula and contains immunological components that may reduce the incidence of sepsis in the immunosuppressed preterm infant. ${ }^{9}$ With the decline in milk banking, ${ }^{10}$ expression may be the only opportunity for an infant to have human milk. Furthermore, the composition of a mother's milk is likely to be more appropriate to the needs of her infant than banked milk, ${ }^{11}$ particularly when pooling or heat treatment ${ }^{12}$ is used.

Prolactin bioactivity has been found to be significantly less in mothers who deliver prematurely, suggesting deficiencies in the initial stimulus for milk production. ${ }^{13}$ Poor initial milk production which then declines is common in these women. ${ }^{4}$ Several studies of term mothers ${ }^{14}{ }^{15}$ suggest that the prolactin response to simultaneous expression compares favourably with infant suckling, although the relation of prolactin release and quantitative milk yield is uncertain. Two studies ${ }^{16}$ on non-breast feeding mothers of preterm infants, in which milk production was compared in women assigned to use sequential single or simultaneous pumping regimens, showed an increase in volume when simultaneous pumping was used. The time taken to express was significantly less in women allocated to simultaneous pumping. ${ }^{16}$ Both studies show variability in milk production between women in all groups. Meier and colleagues ${ }^{18}$ describe the importance of establishing a copious milk yield in the immediate period after preterm delivery, to provide a reserve against the diminishing milk volume that is typical in later weeks of expression.

There is evidence that a decline in milk yield directly signals the mammary gland to curtail production. ${ }^{19}$ Crucially, this will deprive an infant of receiving human milk in the vulnerable period while establishing enteral feeds and may dissuade a mother from continued milk expression. Furthermore, the energy value of expressed milk is variable, ${ }^{20}$ often suggesting 
that only foremilk has been collected, contributing to the sequelae of failure. These include low volumes expressed, maternal frustration, and poor infant growth.

The primary aim of this study was to compare sequential with simultaneous breast pumping on milk volume and energy yield. The secondary aim was to measure the effect of breast massage on milk volume and energy yield. In addition, pumping methods were compared in terms of maternal comfort and efficacy.

\section{Patients and methods}

The study took place on the neonatal unit at North Staffordshire Maternity Hospital between October 1997 and August 1999 with mothers wishing to express breast milk to support their own preterm infants. The unit has an active breast feeding policy, consequently all mothers are provided with an information leaflet on milk expression technique and given the opportunity to view a video made by the researchers covering milk expression and preterm breast feeding. ${ }^{21}$

Mothers wishing to provide breast milk for their infants were approached at 24 hours after delivery and invited to participate in the trial. Parents were given an information sheet explaining the purpose and requirements of the trial. It was also made clear that participation was voluntary and mothers who declined would receive the same high standard of breast feeding support as those in the trial.

Mothers were excluded if they were unable to express a minimum of five times a day before the start of the study or if they had retained products of conception. We were unable to recruit mothers from other areas who were expecting to return to their local hospital within the time frame of the trial, which was to term. Mothers of infants who died during the course of the trial were discouraged from continuing to participate. However, several insisted on attempting to complete the trial stating that they wished to help other mothers in the future.

Signed consent was obtained from mothers electing to take part in the study. Participants were then randomised to either the sequential or simultaneous pumping arm by the opening of a preprepared envelope. As gestational age and parity are known to affect lactation, randomisation was into three groups for gestational age and two groups for parity, giving a total of six groups.

The study period for each participant was four days in total. On two of the days, pumping was preceded by breast massage so that the effects of breast massage could also be determined in the same population. This required randomisation for massage on either days 1, 2 or days 3, 4 using sets of sealed envelopes. On the first day of each 48 hour period, mothers familiarised themselves with the technique of breast massage and data collection. Therefore only the data from the second day of each 48 hour period of the study were analysed.

The trial started on the 4 th day post partum if frequent milk expression had been achieved.
In women with unrelieved engorgement, the trial started after an additional 48 hours of continued expression. During the period of preparation, mothers were taught how to perform breast massage and how to use the milk expression equipment, which was loaned for the duration of the trial. All participants were encouraged to express eight times a day. On each occasion, they were asked to express until milk no longer entered the collection set. In the sequential group, mothers were asked to switch breasts when this point had been reached for the first breast expressed. On the days when massage was used, this took place both before and during each period of expression.

Mothers were given a log book to record the date, time, and duration of each expression. They also recorded the weight of all collection bottles used before expression. The weight after expression was measured, recorded, and validated by the researchers. A capillary sample was taken and stored from each sample for the estimation of fat content. On completion of the trial, women were asked to complete two questionnaires, indicating by an analogue scale their opinion of pump comfort and performance. The questionnaires also contained two descriptive questions: "Have you used a breast pump before"; "how does it compare with the pump you used for this study?". An analogue scale was also used to probe maternal perception of the effectiveness of breast massage when used in conjunction with milk expression. In both questionnaires, mothers were also encouraged to add additional free descriptive comments about milk expression. The women were contacted (telephoned at home if discharged from the unit) to establish the feeding method at term-that is, 37 weeks after conception.

TECHNIQUES AND MEASUREMENT

Breast massage

Breast massage consisted of gentle tactile stimulation of mammary and nipple tissue using a hand action that rolled the knuckles downward over the breast, beginning at the ribs and working towards the areola. ${ }^{22}$ This technique does not involve manual expression of milk.

\section{Milk expression}

The Egnell Ameda Electric Elite pump (Egnell Ameda, Taunton, Somerset, UK) was used because of its ability to create periodic and limited phases of negative pressure and the ease of conversion to the simultaneous pumping mode. Silastic shield inserts were also provided selectively to avoid any disparity between breast and shield size.

\section{Milk yield and fat content}

Milk yield was determined by weight using Salter scales. The accuracy of the scales was checked regularly. The weight before and after expression was always measured on the same scales.

The fat content of expressed milk was determined using the creamatocrit method. ${ }^{24}$ We simplified the method of Lemons et $a l^{24}$ by 
Table 1 Output from a single expression for milk weight and fat for four different combinations of pump modality and breast massage

\begin{tabular}{lllll}
\hline & $\begin{array}{l}\text { Sequential, } \\
\text { non-massage }\end{array}$ & $\begin{array}{l}\text { Sequential, } \\
\text { massage }\end{array}$ & $\begin{array}{l}\text { Simultaneous, } \\
\text { non-massage }\end{array}$ & $\begin{array}{l}\text { Simultaneous, } \\
\text { massage }\end{array}$ \\
\hline Weight $(\mathrm{g})$ & $51(46$ to 56$)$ & $79(73$ to 85$)$ & $88(79$ to 97$)$ & $125(110$ to 140$)$ \\
Fat concentration $(\mathrm{g} / \mathrm{l})$ & $7.1(5.9$ to 8.3$)$ & $6.7(5.7$ to 7.7$)$ & $7.0(5.9$ to 8.1$)$ & $6.8(5.7$ to 7.9$)$ \\
Total fat $(\mathrm{g})$ & $3.1(2.7$ to 3.5$)$ & $4.2(3.8$ to 4.6$)$ & $6.0(5.3$ to 6.7$)$ & $8.0(6.9$ to 9.1$)$ \\
\hline
\end{tabular}

Results are expressed as mean (95\% confidence interval).

sealing the capillary tubes using haematocrit putty (Sartorius Ltd, Epsom, Surrey, UK) rather than a flame, as the former method is more suitable for a clinical area. The creamatocrit method involves drawing a sample of milk into a capillary tube and centrifuging the sample at $5000 \mathrm{rpm}$ for five minutes. Both the column of milk and the liquid fat, which is clearly demarcated at the top of the sample, are measured using an ordinary ruler to the nearest $0.5 \mathrm{~mm}$. The creamatocrit is expressed as percentage of the total milk sample in the tube to the nearest $0.5 \%$. Milk fat concentration is calculated from the creamatocrit formula:

fat $(\mathrm{g} / \mathrm{l})=($ creamatocrit $(\%)-0.59) / 0.146$.

Creamatocrits were measured on freshly expressed milk to prevent fat degradation by milk lipases.

STATISTICAL METHODS

The sample size was calculated at $80 \%$ power and $5 \%$ significance based on an improvement in milk yield of $20 \%$ to $50 \%$. This would require 39 participants for each arm of the study. An interim analysis of complete data on 36 women showed highly significant results in terms of milk yield between the intervention groups. A decision was then made to discontinue recruitment.

Data were analysed using a repeated measures analysis of variance technique (NCSS 97) on the variables mean milk volume and mean fat content. The means were calculated from the total daily volume/amount divided by the number of expressions in that day. The repeat measure comparison was performed between day 2 and 4 of the milk expression data.

ETHICS

The study protocol and information sheet were reviewed and approved by both the local scientific merit committee and the local research ethics committee.

\section{Results}

Between October 1997 and August 1999, 52 mothers gave consent to the trial; 27 were randomised to the sequential group, and 25 to the simultaneous group. There were incomplete data for 16, and they were withdrawn for the following reasons: infant death (four), critically ill infant (five), infant transferred for surgery (two), maternal mastitis (one), and social reasons (four). Nineteen mothers completed the trial in the sequential group, and 17 in the simultaneous group. The statistical design of the study dictated that data could only be analysed from these 36 mothers with complete data.
The sequential pumping group contained seven primiparas and 10 multiparas (six with previous breast feeding experience). There were no significant differences for parity in any of the data (sequential versus simultaneous mean volume, $p=0.62$ ). In addition, this group included three sets of twins (two multiparas, one primipara). The simultaneous pumping group contained five primiparas and 11 multiparas (eight with previous breast feeding experience). In addition, this group included three sets of twins, all born to multiparous women. Overall mean infant birth weight was $1.535 \mathrm{~kg}$ (1.61 kg in the sequential group, and $1.46 \mathrm{~kg}$ in the simultaneous group); mean gestational age was 29.97 weeks (sequential 29.9, simultaneous 30.2). Day 5 post partum was the first day of study for 30 subjects. For six patients, the start of the study was delayed until day 7 because of unrelieved breast engorgement. Unfortunately mothers did not achieve the eight expressions per day that we had planned. However, there was no difference between the mean number of expressions per day between the two study populations (5.2).

Table 1 summarises the results for milk expressed at each session in the four comparison groups along with the fat concentration and total fat content. Differences in milk yield per expression were significant at the $p<0.01$ level between sequential and simultaneous pumping and between massage and nonmassage. The fat concentration of the milk was similar in all four groups. As expected the total fat yield per expression reflected the results for milk yield; these differences were significant at the $\mathrm{p}<0.01$ level for simultaneous pumping compared with sequential pumping but were not significant for massage compared with non-massage.

All the women were asked to express an opinion about equipment efficacy and comfort using an analogue scale. All scores were average (median $=4$, range $0-8$ ), indicating satisfaction with the expression equipment used, although larger sized shields would have produced a better fit for 10 of the mothers. All mothers valued massage, and the median score was 4 (range $0-8)$. Indeed, many mothers were reluctant to drop massage on the non-massage days of the trial if they were allocated to start the trial using massage on days 1 and 2. The mothers allocated to the simultaneous pumping method took much less time for each expression session (about 10-15 minutes as opposed to 25-40 minutes). When mothers were asked for comments, three common themes emerged: an appreciation of the use of massage to expedite milk flow, the time saved by simultaneous pumping, and the need to provide larger milk collection sets.

Data were collected on breast feeding duration from birth until the gestational age reached was equivalent to term (37/40 weeks). In the sequential pumping group, two mothers did not achieve this because of mammary involution, although 15 women were either fully breast feeding or expressing at term; range of weeks of expression or feeding until term was 
5-13. In the simultaneous pumping group, only one mother did not establish breast feeding, and this was because the infant died; range of weeks of expression or feeding until term was $4-11$. Some $68 \%$ of the study population expressed/breast fed for eight weeks until their infants reached term, and $27 \%$ successfully expressed/breast fed for 12 weeks to reach term; $13 \%$ either failed to lactate or suppressed lactation, only providing breast milk initially. There was variability between volumes expressed in all groups studied.

\section{Discussion}

There are few quantitative data to evaluate milk expression protocols for sustaining lactation during the long periods of infant/maternal separation that often accompanies preterm delivery. Insufficient milk volumes followed by declining production are typical. ${ }^{4}$ High levels of prolactin appear to be required to establish lactation, milk macronutrient control, and milk production. ${ }^{23}{ }^{25}$ The presence of prolactin variants is influenced by the stage of mammary development and gestation, ${ }^{13}$ and delays in suckling and milk expression may cause maternal prolactin to decline rapidly during the early postpartum period. ${ }^{23}$ However, the exact relation between prolactin concentration and milk production remains unclear. Studies to evaluate the effect of different methods of pumping on prolactin levels are difficult to interpret because both timing and blood sampling can produce a stress effect and increase prolactin. ${ }^{26}$ Zinnamen et $a l^{14}$ and Zinnamen ${ }^{15}$ studied milk yield, prolactin levels, and oxytocin in mothers allocated to both express and suckle their infants. The results showed an increase in prolactin relative to baseline values of $116 \%$ for the simultaneous pumping method, which equated favourably with infant suckling. The effect of the pumping method on milk yield in mothers of preterm infants was also examined. ${ }^{14}{ }^{15}$ Both studies were designed to evaluate the response to long term pumping (up to 42 days). Both showed increased milk yield when simultaneous pumping was used, and the final study suggested no effect of pumping method on breast feeding outcome. Meier and colleagues ${ }^{18}$ suggest that establishment of an abundant milk yield in the first week of lactation provides a reserve against the diminishing milk volume often found with prolonged pumping. Our study was undertaken to test the hypothesis that simultaneous pumping increases initial milk yield, with an arm added to establish whether tactile breast and nipple massage would provide an impetus to milk ejection and accelerate milk flow. There is strong evidence that tactile stimulation of mammary and nipple tissue facilitates both prolactin and oxytocin responses. Unless an oxytocin response is elicited, facilitating a rise in intramammary pressure, there will be resistance to milk outflow from the alveoli when a breast pump is applied.

This trial clearly shows that simultaneous pumping, with or without massage, is more effective than single pumping in producing an increased volume of milk. Fat production is also significantly higher when simultaneous pumping is used, although this is related to the increase in milk volume rather than an increase in fat concentration of the milk. This may suggest that milk ejection can become conditioned, rather like the micturition reflex, to a range of highly responsive rituals. Massage may be one such ritual. As there is evidence that prolactin levels rise before an oxytocin response, it may be possible that the tactile stimulation of massage may help to compensate for the absence of infant suckling. ${ }^{23}$ There were no statistically significant differences in milk yield between the multiparous and primiparous groups.

This study has found differences in breast feeding outcome when different pumping modalities are used; however, maternal motivation, the provision of expression equipment, an environment conducive to breast feeding, and the skill and enthusiasm of nursing staff are equally important. Indeed the weight expressed per session by simultaneous pumping with massage (125 g, days $2 / 4)$ is in excess of that described as the norm after term delivery in fully breast fed infants $(70 \mathrm{~g}) .^{27}$ This strongly suggests that simultaneous pumping may be able to compensate for initial lactation deficits. Prolactin levels were not evaluated, because it was felt that the need for venepuncture would reduce recruitment. The use of massage was very popular with all study participants. Women felt strongly that expression without preconditioning by massage was more difficult, and many were reluctant to drop the method to complete days $2 / 4$ of the trial. The mean frequency of expression was five times a day for both groups. Open visiting was stated to inhibit more frequent expression.

The expression equipment was well accepted, but breast pump manufacturers should be encouraged to provide collection shields (flanges) of different sizes, as there was disparity between breast and shield sizes in 10 cases. If the funnel aperture is too small, pressure is highest on nipple tissue, ${ }^{28}$ which can cause sore nipples and ineffective drainage. Many of the participants found the demands of the trial very useful, and all appreciated the time spent with the researcher to develop expression skills. The creamatocrit surveillance was especially helpful because it established early specific problems relating to milk flow. Ineffective pumping leads to a low creamatocrit. The clinician and the mother need to evaluate both the quantity and quality of milk produced no later than seven days post partum to determine if intervention is needed so that an adequate supply of milk to feed the preterm infant is available. We think that this technique should be made everyday practice.

In conclusion, this trial has shown conclusively that both milk volume and fat yield can be improved by skilled lactation advice initially, and with the use of simultaneous pumping and breast massage after preterm delivery. The use of simultaneous pumping after preterm delivery may also ensure that the mother's own expressed breast milk is used in the critical period of establishing enteral feeding. 
We are very grateful to Baby Lifeline for funding this project. We would also like to thank all trial participants for the time and effort they took to provide data. We would also like to thank the nursing staff at North Staffordshire Hospital for their help. We
are grateful to Egnell Ameda Ltd for donating collection sets.

\section{Key messages}

- Frequent and efficient milk removal is essential for the continued production of milk

- Breast massage and simultaneous pumping increase the volume of milk expressed in the immediate period after preterm delivery

- Fat content is also higher when simultaneous pumping is used

- Creamatocrit surveillance provides a positive tool to ensure effective milk expression

1 Haggkvist AP. What factors influence the breast feeding prevalence of mothers to premature babies? Proceedings of prevalence of mothers to premature babies? Proceedings of the Child International Conference of

2 Meberg A, Willgraffs, Sande HA. High potential for breast feeding among mothers giving birth to pre-term infants. feeding among mothers giving birth

3 Verronen P. Breastfeeding of low birth weight infants. Acta Paediatr Scand 1985;74:495-9.

4 Hopkinson J, Schanler R, Garza C. Milk production by mothers of premature infants. Pediatrics $1988 ; 81: 815-20$.

5 Meier P, Engstrom J, Mangurten $\mathrm{H}$, et al. Breastfeeding support services in the neonatal intensive care unit. $f$ Obstet Gynecol Neonatal Nurs 1993;22:339-47.

6 Nyquist K, Sjoden P, Ewald U. Mothers'advice about facilitating breastfeeding in a neonatal intensive care unit. $\mathcal{F}$ Hum Lact 1994;10:237-43.

7 Meier P, Brown L. State of the science: breastfeeding for mothers and low birth weight infants. Maternal/Fetal Nursing 1996;11:351-65.

8 Jones E, Spencer SA. Promoting successful breastfeeding for mothers of preterm infants. Professional Care of Mother and mothers of preterm in

9 Narnyanan I, Prakash K, Murthy NS, et al. Randomised controlled trial of effect of raw and Holder pasteurised milk and of formula supplements on the incidence of neonatal infection. Lancet 1984;ii:1111-12.
10 Lucas A. AIDS and milk bank closures. Lancet 1987;i:1092-3.

11 Atkinson SA, Anderson GH, Bryan MH. Human milk: comparison of the nitrogen composition in milk from mothers of premature and full term infants. Am f Clin Nutr 1980;33:811

12 Stein H, Cohen D, Herman AAB, et al. Pooled pasteurised breast milk and untreated own mother's milk in the feeding of very low birth weight babies: a randomised controlled trial. $\mathcal{F}$ Pediatr Gastroenterol Nutr 1986;5:242.

13 Ellis L, Picciano M. Prolactin variants in term and preterm human milk: altered structural characteristic, biological activity and immunoreactivity. Endocrine Regulation 1993;27:181-92.

14 Zinnamen MJ, Jughes V, Queenan JT, et al. Acute prolactin and oxytocin responses and milk yield to infant suckling and artificial methods of expression in lactating women. Pediatrics 1992;89:437-40.

15 Zinnamen MJ. Breastpumps: ensuring mothers' success. Contemporary Obstetrics and Gynecology 1999;32:55-62.

16 Hill P, Aldag J, Chatterton R. The effect of sequential and simultaneous breast pumping on milk volume and prolactin levels: a pilot study. $\mathcal{F}$ Hum Lact 1996;12:193-9.

17 Hill P, Aldag J, Chatterton R. Effects of pumping style on milk production in mothers of non-nursing preterm infants. F Hum Lact 1999;15:209-16.

18 Meier PP, Brown LP, Hurst NM. Breastfeeding the preterm infant. In: Breastfeeding and human lactation. Boston: Jones and Bartlett Publishers, 1999;456.

19 Prentice A, Addey CVP, Wilde CJ. Evidence for local feedback control of human milk secretion, Biochem Soc Trans 1989;17:489-92.

20 Spencer SA, Hull D. Fat content of expressed breast milk: a case for quality control. BMF 1981;282:99-100

21 Jones E, Spencer SA. Breastfeeding the preterm infant [video]. Taunton: Egnell Ameda Ltd, 1996.

22 Spencer SA, Jones E, Dobson J, et al. Breastfeeding: a multimedia learning resource for healthcare professionals. Bradford: Matrix Multimesia, 1998 .

23 Ostrom K. A review of the hormone prolactin during lactation. Progress in Food and Nutrition Science 1990;14:1-4.

24 Lemons JA, Schreiner RL, Gresham EL. Simple method for determining the caloric and fat content of human milk. Pediatrics 1980;66:626.

25 Grosvenor CE, Shyr SW, Goodman GT, et al. Effect of neonatal prolactin deficiency on prepubertal tuberoinfundibular and tuberohypophyseal and dopaminergic neuronal activity. Neuroendocrinology 1986;43:679-85.

26 Nicol CS, Bern HA. On the actions of prolactin among the vertebrates: is there a common denominator? In: Wolsteneds. Lactogenic hormones: Ciba Foundation Symposium. Edinburgh: Churchill-Livingstone, 1972:299-317.

27 Inch S, Fisher C. Breastfeeding: early problems. The Practising Midwife 2000;3:12-15.

28 Johnson CA. An evaluation of breast pumps currently available on the American market. Clin Pediatr 1979;63:597. 